\title{
INVESTIGACIONES
}

\section{Cuestionario de autorregulación del aprendizaje en línea (Online Self-regulated Learning Questionnaire, OSLQ): estudio de validez y fiabilidad de la versión en español}

\author{
Online Self-regulated Learning Questionnaire (OSLQ): \\ study of validity and reliability of the Spanish version
Cecilia Pinto-Santuber ${ }^{a}$, Rodrigo Ortiz-Salgado ${ }^{b}$, Carmen Luz Muñoz Mendoza ${ }^{a}$, Miguel Yáñez Alvarado ${ }^{c}$, Patricia Letelier Sanz ${ }^{a}$ \\ ${ }^{a}$ Universidad del Bío-Bío, Departamento de Enfermería. \\ cepinto@ubiobio.cl,calmunoz@ubiobio.cl,pletelie@ubiobio.cl \\ ${ }^{b}$ Universidad del Bío-Bío, Dirección de Investigación y Creación Artística. \\ rortizsa@ubiobio.cl \\ ${ }^{c}$ Universidad del Bío-Bío, Departamento de Estadística. \\ myanez@ubiobio.cl
}

\begin{abstract}
RESUMEN
En este artículo se presentan los resultados de la validación del cuestionario Aprendizaje Autorregulado en Línea (Online Self-regulated Learning Questionnaire, OSLQ). La adaptación se basó en el método de retrotraducción, a partir de la versión original del instrumento y posterior realización de prueba piloto, con una muestra multidisciplinaria de 238 estudiantes universitarios. Los resultados demuestran una alta consistencia interna de la escala, que presentó un valor 0,90 de alfa de cronbach, por lo tanto, la escala posee una alta confiabilidad y confirma los constructos propuestos por los autores. A través del análisis factorial confirmatorio, se verificó la estructura interna del cuestionario. En conclusión, este instrumento permitirá realizar futuras investigaciones para medir la autorregulación del aprendizaje en estudiantes de la educación superior que utilizan plataformas educativas y apoyar procesos de desarrollo de habilidades cognitivas, motivacional/afectivo y conductual del estudiante.
\end{abstract}

Palabras claves: autorregulación del aprendizaje, enseñanza semipresencial, aprendizaje basado en web, educación superior, plataformas educativas.

\section{ABSTRACT}

In this article the validation results of the Online Self-regulated Learning Questionnaire (OSLQ) are presented. The adaptation was based on a method of retranslation from the original version of the document and the subsequent fulfilment of a pilot test, with a multidisciplinary sample of 238 university students. The results show a high internal consistency of the scale, which presented a 0.90 Cronbach alpha value, and therefore, the scale is highly reliable and confirms the constructs proposed by the authors. Through the use of confirmatory factorial analysis, the internal structure of the questionnaire was verified. In conclusion, this document will permit future investigations to be carried out in order to measure self-regulated learning in higher education students that use educational platforms, and it will support the development processes of cognitive, motivational/emotional and behavioural abilities of the student.

Key words: self-regulated learning, blended learning, E-learning, higher education, educational platforms. 
Estudios Pedagógicos XLVI N² 2: 251-266, 2020

CUESTIONARIO DE AUTORREGULACIÓN DEL APRENDIZAJE EN LÍNEA (ONLINE SELF-REGULATED LEARNING QUESTIONNAIRE, OSLQ): ESTUDIO DE VALIDEZ Y FIABILIDAD DE LA VERSIÓN EN ESPAÑOL

\section{INTRODUCCIÓN}

El desarrollo tecnológico en las últimas décadas ha tenido un crecimiento acelerado y un rol primordial en los procesos educativos. Como una forma de adaptarse a estos cambios y a las necesidades del alumnado, las plataformas educativas han sido incorporadas por las universidades de forma intensiva para complementar las clases presenciales tradicionales.

Esta nueva modalidad de enseñanza se ha denominado como aprendizaje mixto o combinado (blended learning), que, debido a su masificación a nivel mundial, ha sido denominado como "el nuevo modelo tradicional" o "la nueva normalidad" en la implementación de cursos de Educación Superior (Ross y Gage, 2006; Norberg, Dziuban y Moskal, 2011; Graham, Woodfield y Buckey, 2013).

En Chile, debido a las políticas educativas estatales basadas en el public managment (gerenciamiento público) y el cumplimiento de indicadores y criterios de aseguramiento de la calidad, las universidades implementaron masivamente el uso de plataformas, las cuales, en general, tendieron inicialmente a funcionar a manera de repositorio de recursos digitales de las clases presenciales, más que funcionar con un diseño instructivo semipresencial. Sin embargo, existieron variadas experiencias de innovaciones con el uso de estas herramientas digitales, y para dar cuenta de su desarrollo se publicaron múltiples estudios en los últimos años que, de acuerdo a Jaramillo (2015), tendieron a mostrar en investigaciones empíricas (62\%) y sistematizar experiencias (28\%), ocupando una menor proporción el desarrollo de perspectivas teóricas sobre aprendizaje en contextos virtuales $(10 \%)$.

De acuerdo a lo que señala la literatura internacional acerca de la modalidad de enseñanza mixta, los cursos con apoyo de plataformas educativas se caracterizan por su ubicuidad, eliminan la limitación de espacio-tiempo, materiales físicos y en gran medida, el control sobre cuándo y cómo estudiar, además, proporcionan libertad de moverse sin restricciones de un tema a otro (McManus, 2000; Cunningham y Billingsley 2003; Moran 2012; Vaughan, Cleveland-Innes y Garrison, 2013). Sin embargo, a pesar de las ventajas que proporcionan las plataformas educativas, se espera que los estudiantes desarrollen habilidades de estudio complementarias, relacionadas con la autonomía y el compromiso activo para que obtengan resultados de aprendizaje efectivos, dada la menor interacción con instructores o compañeros (Ally, 2004; Lee y Choi, 2011). En este sentido, existe bastante evidencia empírica que señala que a medida que el entorno de aprendizaje en línea se caracteriza por la autonomía, la autorregulación se convierte en un factor crítico para obtener aprendizajes profundos. Estas investigaciones indican que los estudiantes que son más capaces de regular su aprendizaje rinden mejor que los estudiantes que son menos capaces de regularlo (Schunk y Zimmerman, 1998; Schunk, 2001).

Pero la autorregulación del proceso de aprendizaje no es una propiedad inherente de los estudiantes universitarios, sino más bien es una dificultad, que repercute directamente en su desempeño académico. No obstante, los procesos de autorregulación son educables, es decir, se pueden modificar y mejorar, permitiendo incluso conducir a incrementar el rendimiento y la motivación de los alumnos, ya que posibilita tomar decisiones para mejorar la capacidad de monitorear, regular y ajustar sus habilidades (Allgood, Risko, Alvarez \& Fairbanks, 2000). En este aspecto radica la importancia de medir la capacidad de autorregulación de los estudiantes, ya que proporciona información valiosa para mejorar sus desempeños educativos. 
La conceptualización y análisis de los procesos y actividades relacionados con las capacidades de autorregulación del aprendizaje tienen un desarrollo consolidado hace varias décadas por la psicología educativa. Zimmerman (1990) y Pintrich (2000), los principales referentes en área, lograron definir que el individuo que aprende, se percibe dentro de un ambiente cambiante que lo obliga a realizar constantes esfuerzos metacognitivos de evaluación y realimentación para ajustar sus conductas y estrategias, relacionadas con el establecimiento de metas, estrategias para afrontar las tareas, capacidad de autoevaluación del proceso, manejo del tiempo, regulación del ambiente de aprendizaje y la capacidad de pedir ayuda (Zimmerman 1990; Schunk y Zimmerman 1994; Zimmerman 2001; Azevedo 2007).

Para medir la autorregulación de los estudiantes en entornos de aprendizaje en contextos virtuales, Barnard, Lan, To, Osland y Lai (2009) desarrollaron un cuestionario que incorpora las dimensiones y componentes conceptuales desarrollados por Pintrich y Zimmerman, el cual denominaron "Online Self-regulated Learning Questionnaire" (aprendizaje autorregulado en línea, OSLQ), que tiene la intensión de proporcionar un medio para evaluar las habilidades de aprendizaje de autorregulación de los estudiantes, tanto en los entornos totalmente virtuales como en aquellos semipresenciales.

Desde que se creó, este instrumento ha sido adaptado y utilizado en diferentes contextos y culturas, dando cuenta de su fiabilidad y validez en países como, Estados Unidos, Reino Unido, Rusia, China, Países Bajos, y Turquía; además, de demostrar ser confiables tanto en contextos virtuales como híbridos, obteniendo resultados rigurosos publicados en las principales revistas científicas del mundo (Barnard et al., 2009, 2010a; Zolotykhin, 2015; Onah y Sinclair 2016; Martinez et al., 2017; Fung et al., 2018; Kilis y Yildirim, 2018).

A nivel hispanoamericano pudimos constatar que este cuestionario no ha sido validado, y que, además, no se cuenta con otros instrumentos en español que midan las capacidades autorreguladoras del aprendizaje en un contexto en línea mixto (de acuerdo con la búsqueda en revistas de bases de datos WOS, Scopus y Scielo).

El presente estudio tiene como propósito mostrar la validación del cuestionario OSLQ realizada con estudiantes de una universidad chilena, y tiene como objetivo contribuir a la disposición de un instrumento que entrega herramientas conceptuales que promueva la reflexión y análisis de los procesos de enseñanza con apoyo de plataformas virtuales en la Educación Superior, generando datos de manera masiva, ante un contexto investigativo regional con una escasa profundización teórica, tanto a nivel general de tecnologías de información (TIC), como específico en el ámbito b-learning.

Para la adaptación del cuestionario se aplicó, en primer lugar, la metodología de retrotraducción o back traslation, lo que permitió obtener una primera versión en español. Posteriormente fue validada a través de expertos, para luego, realizar un análisis factorial confirmatorio a través de una prueba piloto conformada por una muestra de 238 alumnos de una universidad estatal chilena. Los resultados mostraron una alta confiabilidad, con indicadores adecuados por ítem, dimensión y constructo, además de demostrar medidas de bondad del ajuste acordes a la literatura consultada, lo que permite sustentar que este cuestionario puede comenzar a aplicarse con fines investigativos y de desarrollo académico en estudiantes universitarios chilenos. 
Estudios Pedagógicos XLVI Nº 2: 251-266, 2020

CUESTIONARIO DE AUTORREGULACIÓN DEL APRENDIZAJE EN LÍNEA (ONLINE SELF-REGULATED LEARNING

QUESTIONNAIRE, OSLQ): ESTUDIO DE VALIDEZY FIABILIDAD DE LA VERSIÓN EN ESPAÑOL

\section{DESARROLLO DE UN CUESTIONARIO PARA EVALUAR LA AUTORREGULACIÓN DEL APRENDIZAJE EN CONTEXTOS VIRTUALES}

La relación entre autorregulación del aprendizaje y rendimiento académico ha sido desarrollada por diferentes enfoques y estudios, pero sin lugar a duda, los modelos de Pintrich y Zimmerman, son los que han sintetizado de mejor forma los avances de esta área.

Dentro de todos ellos, estos autores destacan el modelo de Pintrich (2000), como uno de los intentos de síntesis más importantes realizados sobre los diferentes procesos y actividades que ayudan a acrecentar la autorregulación del aprendizaje. Pintrich propuso un marco teórico basado en una perspectiva sociocognitiva, que considera los procesos reguladores del aprendizaje organizados en cuatro fases: a) la planificación; b) la autoobservación (self-monitoring); c) el control; y d) la evaluación, que representan una secuencia general por donde el alumno avanza a medida que realiza la tarea, pero no están jerárquica o linealmente estructuradas. A su vez, dentro de cada una de ellas, las actividades de autorregulación se enmarcan en cuatro áreas: la cognitiva, la motivacional/afectiva, la comportamental y la contextual (Pintrich, 2000). Para medir la autorregulación de los estudiantes en el entorno de aprendizaje en línea, Lan, Bremer, Stevens y Mullen (2004) desarrollaron un Cuestionario de Aprendizaje Autorregulado en Línea (OSLQ) el cual se desarrolló para reflejar una concepción multidimensional del aprendizaje autorregulado, derivado del marco teórico desarrollado por Zimmerman (1998). Posteriormente Barnard et al., (2009) lo adaptaron a una versión abreviada de 24 ítems, conformado por seis subescalas. Los factores y el número de elementos que reflejan estas subescalas son :ES con 5 artículos implica "seleccionar o crear ajustes efectivos para el aprendizaje", GS con 4 artículos significa "especificar acciones o resultados previstos", TS con 4 artículos significa "analizar tareas e identificar métodos específicos y ventajosos para aprender", TM con 4 artículos, se refiere a "estimar y presupuestar el uso del tiempo", HS con 3 artículos significa "elegir modelos específicos, maestros o libros para ayudarse a uno mismo a aprender", y finalmente SE con 4 artículos significa "establecer estándares y usarlos para el juicio propio" (Zimmerman, 1998). El cuestionario abreviado contiene un formato de respuesta tipo Likert de 5 puntos, con valores que van desde muy de acuerdo (5) a muy en desacuerdo. Las puntuaciones más altas en esta escala indican una mejor autorregulación en el aprendizaje en línea por los estudiantes.

El OSLQ se ha validado y encontrado confiable (Barnard et al., 2009). La validez se verificó con un análisis factorial confirmatorio y los índices de ajuste se consideraron aceptables. Los resultados evidencian la validez de construcción del instrumento para los estudiantes tanto en entornos de aprendizaje combinado como en línea. La primera versión del instrumento fue sometida a una validación con 2 muestras, una con estudiantes de curso b-learning (n:434) y otro con estudiantes e-learning (n: 628) de diferentes universidades estadounidenses, y en ambos casos demostraron una alta consistencia interna, con alfa de Cronbach de 0,90 y 0,92 respectivamente. La consistencia interna por dimensión demostró resultados satisfactorios, con valores que fluctuaron entre 0,67 y 0,96 (Barnard et al., 2009). Posteriormente, el mismo núcleo de investigación implementó dos investigaciones. La primera experiencia se enfocó en estudiantes virtuales nóveles, que a través de un análisis cuasi-experimental pudieron determinar que no hay una diferencia significativa de las habilidades de autorregulación a lo largo del tiempo en alumnos que se ven enfrentados por primera vez a un entorno en línea (Barnard et al., 2010a). La segunda investigación 
realizada tuvo como objetivo determinar niveles de autorregulación del aprendizaje, que fueron aplicadas en estudiantes de educación superior norteamericanos, identificando 5 perfiles distintos de aprendizaje autorregulado (Barnard, Osland y Lan 2010b).

Con la finalidad de complementar estas primeras experiencias de validación del cuestionario, se amplió la búsqueda a estudios desarrollados en contextos similares que explicitaran la metodología empleada y continuarán con los lineamientos del OLSQ. Se exploraron las bases de datos Web of Science, Scopus y Scielo. En la siguiente tabla se puede observar que el cuestionario ha sido aplicado en estudios de distinta naturaleza, que fijaron como objetivos medir la capacidad de autorregulación en diferentes entornos de enseñanza y de diversas regiones del mundo, siendo el propósito de algunos de ellos la validación de OSLQ, con buenos resultados de confiabilidad.

Tabla 1. Estudios que utilizaron el OSLQ

\begin{tabular}{|c|c|c|c|c|}
\hline Estudio & Propósito & Tipo de estudio & Muestra & Alfa de Cronbach \\
\hline \multirow[t]{2}{*}{$\begin{array}{l}\text { (Barnard et al., } \\
\text { 2009) }\end{array}$} & \multirow{2}{*}{$\begin{array}{l}\text { Desarrollar el Cuestionario de } \\
\text { aprendizaje autorregulado en } \\
\text { línea y mixto (OSLQ), en } \\
\text { estudiantes universitarios } \\
\text { norteamericanos }\end{array}$} & Transeccional. & $\begin{array}{l}\mathrm{N}=434 \\
\text { estudiantes } \\
\text { (b-learning) }\end{array}$ & 0,90 \\
\hline & & & $\begin{array}{l}\mathrm{N}=628 \\
\text { estudiantes } \\
\text { (online) }\end{array}$ & 0,92 \\
\hline $\begin{array}{l}\text { (Barnard et al., } \\
\text { 2010a) }\end{array}$ & $\begin{array}{lr}\text { Determinar habilidades } & \text { de } \\
\text { autorregulación de los } \\
\text { estudiantes universitarios } \\
\text { norteamericanos en } & \text { su } \\
\text { primera experiencia } & \text { de } \\
\text { aprendizaje en línea. }\end{array}$ & $\begin{array}{l}\text { Cuasi- } \\
\text { experimental }\end{array}$ & $\begin{array}{l}\mathrm{N}=209 \\
\text { estudiantes } \\
\text { online } \\
\text { (Pretest y } \\
\text { Postest) }\end{array}$ & \\
\hline \multirow[t]{2}{*}{$\begin{array}{l}\text { (Barnard et al., } \\
\text { 2010b) }\end{array}$} & \multirow{2}{*}{\begin{tabular}{|lr} 
Examinar si & existen perfiles \\
de habilidades y estrategias de \\
aprendizaje & autorreguladas \\
entre & estudiantes \\
universitarios & de \\
Norteamérica. & \\
\end{tabular}} & \multirow[t]{2}{*}{ Transeccional } & $\begin{array}{l}\mathrm{N}=279 \\
\text { estudiantes } \\
\text { online }\end{array}$ & 0,90 \\
\hline & & & $\begin{array}{l}\mathrm{N}=197 \\
\text { estudiantes } \\
\text { online }\end{array}$ & 0,92 \\
\hline (Kirmizi, 2014) & $\begin{array}{l}\text { Comparar las fortalezas del } \\
\text { aprendizaje autorregulado en } \\
\text { estudiantes regulares, a } \\
\text { distancia y vespertinos de } \\
\text { Lengua y Literatura Inglesa, } \\
\text { en una Universidad de } \\
\text { Turquía }\end{array}$ & Transeccional & $\begin{array}{l}\mathrm{N}=237 \\
\text { estudiantes } \\
\text { online }\end{array}$ & \\
\hline $\begin{array}{l}\text { (Tabuenca, } \\
\text { Kalz, Drachsler } \\
\text { y Specht, 2015) }\end{array}$ & $\begin{array}{l}\text { Efectos del monitoreo del } \\
\text { tiempo dedicado a aprender, } \\
\text { utilizando un dispositivo } \\
\text { móvil en estudiantes de } \\
\text { postgrado de los Países Bajos }\end{array}$ & Longitudinal & $\mathrm{N}=36$ & \\
\hline
\end{tabular}


Estudios Pedagógicos XLVI N 2: 251-266, 2020

CUESTIONARIO DE AUTORREGULACIÓN DEL APRENDIZAJE EN LÍNEA (ONLINE SELF-REGULATED LEARNING QUESTIONNAIRE, OSLQ): ESTUDIO DE VALIDEZ Y FIABILIDAD DE LA VERSIÓN EN ESPAÑOL

\begin{tabular}{|c|c|c|c|c|}
\hline $\begin{array}{l}\text { (Lai y Hwang, } \\
\text { 2016) }\end{array}$ & $\begin{array}{ll}\text { Estudio cuasiexperimental } \\
\text { comparativo entre una } \\
\text { propuesta de aula flexible } \\
\text { autorregulada y aula invertida } \\
\text { convencional (fliped), en } \\
\text { estudiantes chinos de } \\
\text { primaria. }\end{array}$ & $\begin{array}{l}\text { Cuasi- } \\
\text { experimental }\end{array}$ & $\begin{array}{l}\text { Grupo } \\
\text { experi- } \\
\text { mental: } 20 ; \\
\text { grupo de } \\
\text { control: } 24 \\
\text { estudiantes }\end{array}$ & \\
\hline $\begin{array}{l}\text { (Kintu y Zhu, } \\
\text { 2016) }\end{array}$ & $\begin{array}{l}\text { Determinar si las } \\
\text { características, el contexto y } \\
\text { el diseño instructivo } \\
\text { combinado, son factores } \\
\text { relevantes en los resultados de } \\
\text { aprendizaje de estudiantes } \\
\text { secundarios de Uganda }\end{array}$ & $\begin{array}{l}\text { Transeccional } \\
\text { - evaluativo }\end{array}$ & $\begin{array}{l}\mathrm{N}=270 \\
\text { estudiantes } \\
\text { b-learning }\end{array}$ & \\
\hline $\begin{array}{l}\text { (Onah y } \\
\text { Sinclair, 2016) }\end{array}$ & $\begin{array}{l}\text { Identificar percepción sobre } \\
\text { autorregulación del } \\
\text { aprendizaje en Modalidad } \\
\text { B-learning con plataforma } \\
\text { MOOC, en estudiantes de } \\
\text { pregrado del Reino Unido }\end{array}$ & $\begin{array}{l}\text { Estudio mixto } \\
\text { (cualitativo y } \\
\text { cuantitativo) }\end{array}$ & $\begin{array}{l}\mathrm{N}=107 \\
\text { estudiantes } \\
\text { b-learning }\end{array}$ & \\
\hline $\begin{array}{l}\text { (Martínez- } \\
\text { López, Yot, } \\
\text { Tuovila y } \\
\text { Perera- } \\
\text { Rodríguez, } \\
\text { 2017) }\end{array}$ & $\begin{array}{l}\text { Validar cuestionario OSLQ } \\
\text { para cursos MOOC en } \\
\text { contexto ruso }\end{array}$ & $\begin{array}{l}\text { Prueba piloto y } \\
\text { escala de } \\
\text { comprensión } \\
\text { verbal }\end{array}$ & $\mathrm{N}=45$ & 0,95 \\
\hline $\begin{array}{l}\text { (Fung, Yuen y } \\
\text { Yuen, 2018) }\end{array}$ & $\begin{array}{l}\text { Validar cuestionario OSLQ } \\
\text { para contexto chino, análisis } \\
\text { comparado de estudiantes } \\
\text { regulares y con talento } \\
\text { matemático }\end{array}$ & Transeccional & $\begin{array}{l}\mathrm{N}=412 \\
\text { (regulares) } \\
\text { y } 374 \text { con } \\
\text { talento } \\
\text { matemático }\end{array}$ & 0,94 \\
\hline $\begin{array}{l}\text { (Kilis y } \\
\text { Yildirim, 2018) }\end{array}$ & 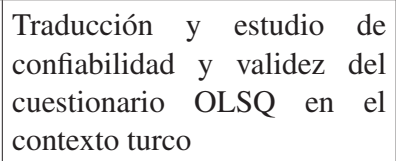 & Transeccional & $\mathrm{N}=321$ & 0,95 \\
\hline
\end{tabular}

Fuente. Elaboración propia.

En la tabla 2 se observan estudios que calcularon medida de bondad de ajuste del análisis factorial confirmatorio, que permitirá evaluar si los resultados logrados en este estudio son equivalentes a los presentados en la literatura. 
Tabla 2. Cuadro comparativo de estudios consultados que realizaron análisis factorial confirmatorio

\begin{tabular}{|c|c|c|c|}
\hline Estudio & (Barnard et al., 2009) & (Fung et al., 2018) & (Kilis y Yildirim, 2018) \\
\hline X2/GL & 3,08 & 1,55 & 2,45 \\
\hline RMSEA & 0,04 & 0,04 & 0,06 \\
\hline NNFI & 0,95 & 0,96 & 0,8 \\
\hline CFI & 0,96 & 0,98 & 0,9 \\
\hline
\end{tabular}

Fuente. Elaboración propia.

Con la finalidad de comparar los resultados obtenidos en este estudio con otras investigaciones que han utilizado y validado el instrumento OSLQ en diferentes partes del mundo, se presenta la siguiente tabla 3 con resultados de consistencia interna por subescala.

Tabla 3. Análisis comparativo de investigaciones que han analizado la fiabilidad por consistencia interna por subescala

\begin{tabular}{|l|c|c|c|c|c|c|}
\hline & \multicolumn{5}{|c|}{ Alfa de Cronbach por Subescalas } \\
\hline \multicolumn{1}{|c|}{ Estudio } & $\begin{array}{c}\text { Estructura } \\
\text { del Ambiente }\end{array}$ & $\begin{array}{c}\text { Establecimiento } \\
\text { de Metas }\end{array}$ & $\begin{array}{c}\text { Gestión del } \\
\text { Tiempo }\end{array}$ & $\begin{array}{c}\text { Búsqueda } \\
\text { de Ayuda }\end{array}$ & $\begin{array}{c}\text { Estrategias } \\
\text { de Tareas }\end{array}$ & $\begin{array}{c}\text { Autoeva- } \\
\text { luación }\end{array}$ \\
\hline (Barnard et al., 2009) & 0,90 & 0,86 & 0,78 & 0,69 & 0,67 & 0,78 \\
\hline & 0,92 & 0,95 & 0,87 & 0,96 & 0,93 & 0,94 \\
\hline (Barnard et al., 2010a) & 0,75 & 0,89 & 0,76 & 0,75 & 0,76 & 0,72 \\
\hline & 0,79 & 0,93 & 0,73 & 0,74 & 0,77 & 0,73 \\
\hline (Barnard et al., 2010b) & 0,92 & 0,88 & 0,91 & 0,92 & 0,85 & 0,89 \\
\hline & 0,92 & 0,95 & 0,89 & 0,88 & 0,92 & 0,94 \\
\hline (Kirmizi, 2014) & 0,80 & 0,76 & 0,71 & 0,67 & 0,60 & 0,83 \\
\hline (Tabuenca et al., 2015) & 0,78 & 0,83 & 0,76 & 0,69 & 0,41 & 0,59 \\
\hline (Lai y Hwang, 2016) & 0,95 & 0,92 & 0,93 & 0,87 & 0,96 & 0,94 \\
\hline (Kintu y Zhu, 2016) & 0,77 & 0,85 & 0,68 & 0,63 & 0,72 & 0,78 \\
\hline (Martínez-López et al., 2017) & 0,91 & 0,8 & 0,96 & 0,96 & 0,92 & 0,84 \\
\hline (Fung et al., 2018) & 0,81 & 0,87 & 0,81 & 0,79 & 0,77 & 0,87 \\
\hline (Kilis y Yildirim, 2018) & 0,87 & 0,79 & 0,75 & 0,71 & 0,67 & 0,81 \\
\hline
\end{tabular}

Fuente: Elaboración propia. 
Estudios Pedagógicos XLVI N² 2: 251-266, 2020

CUESTIONARIO DE AUTORREGULACIÓN DEL APRENDIZAJE EN LÍNEA (ONLINE SELF-REGULATED LEARNING QUESTIONNAIRE, OSLQ): ESTUDIO DE VALIDEZY FIABILIDAD DE LA VERSIÓN EN ESPAÑOL

\section{METODOLOGÍA}

Como se mencionó anteriormente, este estudio tuvo como objetivo traducir y validar el Cuestionario de Aprendizaje Autorregulado en Línea (OSLQ) creado por Barnard et al., 2009. Para esto se siguió el método de retrotraducción o backtraslation, que incluyeron diferentes instancias de adecuación idiomática y contextual, para luego desarrollar la validación estadística a través de prueba piloto. A continuación, se describe en detalle cada uno de los métodos implementados.

\subsection{PROCESO DE TRADUCCIÓN Y RETROTRADUCCIÓN}

Se contactó con los autores de la versión original, y se solicitó tanto el instrumento en inglés como la autorización para su adaptación al español. Siguiendo la propuesta de Bombardier y Beaton; Leplège y Verdier, para la adaptación de cuestionarios, basada en el método de traducciones y retrotraducciones, el cuestionario original fue traducido al español por 2 personas bilingües, quienes trabajaron de manera independiente (Bombardier y Beaton, 1993; Leplège y Verdier, 1995). El cuestionario entregado incluía una breve descripción de las dimensiones que lo compone y se les solicitó que cada ítem debía describir una conducta referida a la autorregulación; las traducciones debían ser semánticas y no literales, enfatizándose la equivalencia conceptual e intentando dar sentido a los ítems en español, pero sin variar el significado o la intención de cada ítem de la versión original.

El primer proceso de traducción dio lugar a 2 versiones en español, que fueron evaluadas cualitativamente por el equipo investigador de forma conjunta. Se valoró la equivalencia conceptual y la claridad de su redacción, hasta alcanzar un acuerdo para llegar a una primera versión.

Este prototipo fue sometido a un estudio de interpretabilidad de ítems a modo de prueba piloto, sobre una muestra de 5 estudiantes de educación superior del área de salud, que habían cursado asignaturas b-learning y que presentaban características sociodemográficas heterogéneas. Luego que los estudiantes respondieran de forma individual el cuestionario, se realizaron consultas siguiendo lineamientos de una entrevista grupal cualitativa, con relación al formulario en general y de forma específica por cada ítem. No hubo sugerencias significativas con relación a la estructura, fluidez o consistencia de las preguntas, sin embargo, se sugirió cambiar la redacción de 2 ítems: eliminar referencias relacionadas con periodos de tiempo de estudio, y actualizar conceptos de herramientas virtuales que se utilizan actualmente para comunicarse con los instructores de los cursos (redes sociales), que no formaban parte de la primera versión del cuestionario del año 2008.

Con el resultado de esta segunda versión, se solicitó a 2 profesores bilingües que realizaran la retrotraducción en forma independiente. Posteriormente, 2 docentes bilingües diferentes a los anteriores evaluaron cuantitativamente, en una escala de 1 a 5 , la equivalencia sintáctica y semántica entre el cuestionario original y la retrotraducción, obteniendo la versión que sería aplicada en la prueba estadística piloto.

\subsection{CARACTERÍSTICA DE LA MUESTRA}

El diseño del estudio fue descriptivo de corte transversal, sobre una muestra no probabilística de estudiantes pertenecientes a las carreras de Ingeniería Civil en Informática, Enfermería, 
Fonoaudiología, Pedagogía en Educación General Básica y Pedagogía en Educación Parvularia, de cursos de niveles superiores de una universidad estatal chilena, que habían experimentado asignaturas presenciales con apoyo de plataformas virtuales (b-learning). La recogida de datos la realizaron encuestadores previamente entrenados, durante el mes de noviembre del año 2017. Además, se complementó el Cuestionario de Aprendizaje Autorregulado en Línea, con variables sociodemográficas relevantes al contexto: edad y género; variables académicas: carrera, frecuencia de acceso a la(s) plataforma(s) educativas, lugar desde donde accede y tipo de herramienta tecnológica que utiliza para acceder a la plataforma educativa.

La muestra finalmente quedó conformada por 238 estudiantes, con una media de edad de 21,35 $\pm 2,61$ años, y de los que el 73,9\% eran mujeres. El mayor porcentaje de estudiantes correspondió a la carrera de Enfermería con un 47,1\%. La plataforma más utilizada fue Adecca con un 53,4\% y el 60,1\% de los participantes declara que accede a las plataformas desde el computador de su casa.

\subsection{EVALUACIÓN DE LAS CARACTERÍSTICAS PSICOMÉTRICAS DEL INSTRUMENTO}

Para evaluar la fiabilidad por consistencia interna de la puntuación global y de cada subescala del cuestionario, se calculó el coeficiente alfa de Cronbach. De acuerdo con las recomendaciones de Nunally para estudios de fiabilidad, en el área de ciencias sociales, se consideró como valor aceptable un coeficiente igual o mayor a 0,70 (Nunally, 1978). Para establecer la validez de constructo, se realizó un análisis factorial confirmatorio de orden superior, reportando los siguientes estadísticos de bondad del ajuste del modelo: el estadístico chi-cuadrado $\left(\chi^{2}\right)$; el estadístico chi-cuadrado normado $\left(\chi^{2} / g . l\right)$; la raíz del error cuadrático medio de aproximación (Root Mean Square Error of Approximation, RMSEA); el índice de ajuste no normado (Non Normed Fit Index, NNFI); y el índice de ajuste comparativo (Comparative Fit Index, $C F I$ ). Finalmente, para estudiar la interrelación de las preguntas que conforman las dimensiones en relación al puntaje de la dimensión y del total del puntaje, se utilizaron coeficientes de correlación de Pearson. Los análisis fueron realizados con los programas estadísticos SPSS versión 20 y LISREL versión 8.54 (Jöreskog y Sörbom, 2003).

\section{RESULTADOS}

\subsection{ANÁLISIS DE FIABILIDAD POR CONSISTENCIA INTERNA}

En la tabla 4 se presentan los coeficientes alfa de Cronbach de cada una de las 6 subescalas y del cuestionario total. 
Tabla 4. Fiabilidad por consistencia interna

\begin{tabular}{|l|c|}
\hline Subescala & Alfa de Cronbach \\
\hline Estructura del Ambiente & 0,71 \\
\hline Establecimiento de Metas & 0,69 \\
\hline Gestión del Tiempo & 0,76 \\
\hline Búsqueda de Ayuda & 0,67 \\
\hline Estrategias de Tareas & 0,71 \\
\hline Autoevaluación & 0,77 \\
\hline Puntuación Global & $\mathbf{0 , 9 0}$ \\
\hline
\end{tabular}

Fuente. Elaboración propia.

Como se puede observar, la consistencia interna global del cuestionario es muy buena $(0,90)$, mientras que en cuatro de las seis dimensiones los valores son satisfactorios, únicamente se obtiene un coeficiente más bajo, en las subescalas "Búsqueda de Ayuda" $(0,67)$ y "Establecimiento de Metas" $(0,69)$.

\subsection{VALIDEZ DEL CONSTRUCTO: ANÁLISIS FACTORIAL CONFIRMATORIO}

Para el análisis factorial confirmatorio, el estadístico chi-cuadrado fue significativo, indicando que el modelo se ajusta a los datos. El valor del estadístico es de 3,5 el cual al ser menor que 5 indica que el modelo es aceptable (Marsh y Hocevar, 1985). El estadístico RMSE fue de 0,073, indica un modelo aceptable al ser menor que 0,08 (MacCallum, Browne y Sugawara, 1996). Los valores de los índices NNFI y CFI fueron ambos de 0.98, siendo estos indicativos de buen ajuste (Hu y Bentler, 1999). De esta forma, el modelo estimaría bien los datos.

La tabla 5 muestra los coeficientes de trayectorias estandarizados desde el constructo latente al ítem. Las trayectorias en el modelo fueron todas significativas $(\mathrm{p}<0,05)$ con valores en el rango 0,13 a 0,82 . Los resultados indican evidencia de validez de constructo. Dentro del constructo "Establecimiento de Metas", el ítem 5 (P5) es el único que no presenta un peso importante, pero no altera las propiedades de la dimensión. 
Tabla 5. Coeficientes de trayectorias estandarizados

\begin{tabular}{|l|c|l|c|}
\hline \multicolumn{1}{|c|}{ Trayectoria } & Coef. Stand. & \multicolumn{1}{|c|}{ Trayectoria } & Coef. Stand. \\
\hline Establecimiento de metas $\rightarrow$ P1 & 0,66 & Gestión del tiempo $\rightarrow$ P14 & 0,62 \\
Establecimiento de metas $\rightarrow$ P2 & 0,73 & Gestión del tiempo $\rightarrow$ P15 & 0,82 \\
Establecimiento de metas $\rightarrow$ P3 & 0,79 & Gestión del tiempo $\rightarrow$ P16 & 0,81 \\
Establecimiento de metas $\rightarrow$ P4 & 0,81 & Búsqueda de ayuda $\rightarrow$ P17 & 0,60 \\
Establecimiento de metas $\rightarrow$ P5 & 0,13 & Búsqueda de ayuda $\rightarrow$ P18 & 0,77 \\
Estructura del ambiente $\rightarrow$ P6 & 0,70 & Búsqueda de ayuda $\rightarrow$ P19 & 0,68 \\
Estructura del ambiente $\rightarrow$ P7 & 0,66 & Búsqueda de ayuda $\rightarrow$ P20 & 0,54 \\
Estructura del ambiente $\rightarrow$ P8 & 0,76 & Autoevaluación $\rightarrow$ P21 & 0,70 \\
Estructura del ambiente $\rightarrow$ P9 & 0,76 & Autoevaluación $\rightarrow$ P22 & 0,79 \\
Estrategias de tareas $\rightarrow$ P10 & 0,64 & Autoevaluación $\rightarrow$ P23 & 0,65 \\
Estrategias de tareas $\rightarrow$ P11 & 0,65 & Autoevaluación $\rightarrow$ P24 & 0,72 \\
Estrategias de tareas $\rightarrow$ P12 & 0,69 & & \\
Estrategias de tareas $\rightarrow$ P13 & 0,64 & & \\
\hline
\end{tabular}

Fuente. Elaboración propia.

El modelo factorial confirmatorio de orden superior representado en la Figura 1, evidencia que la "Autorregulación" afecta fuertemente a las "Estrategias de Tareas", la "Gestión del Tiempo" y el "Establecimiento de Metas", con coeficientes estandarizados de $0,90,0,86$ y 0,84 , respectivamente, todos significativos al nivel 0,05. En menor medida, la Autorregulación en Línea se relaciona con la "Autoevaluación" $(0,78)$, la "Estructura del Ambiente" $(0,71)$ y la "Búsqueda de Ayuda" $(0,70)$.

Figura 1. Diagrama de trayectorias OSLQ versión es español.

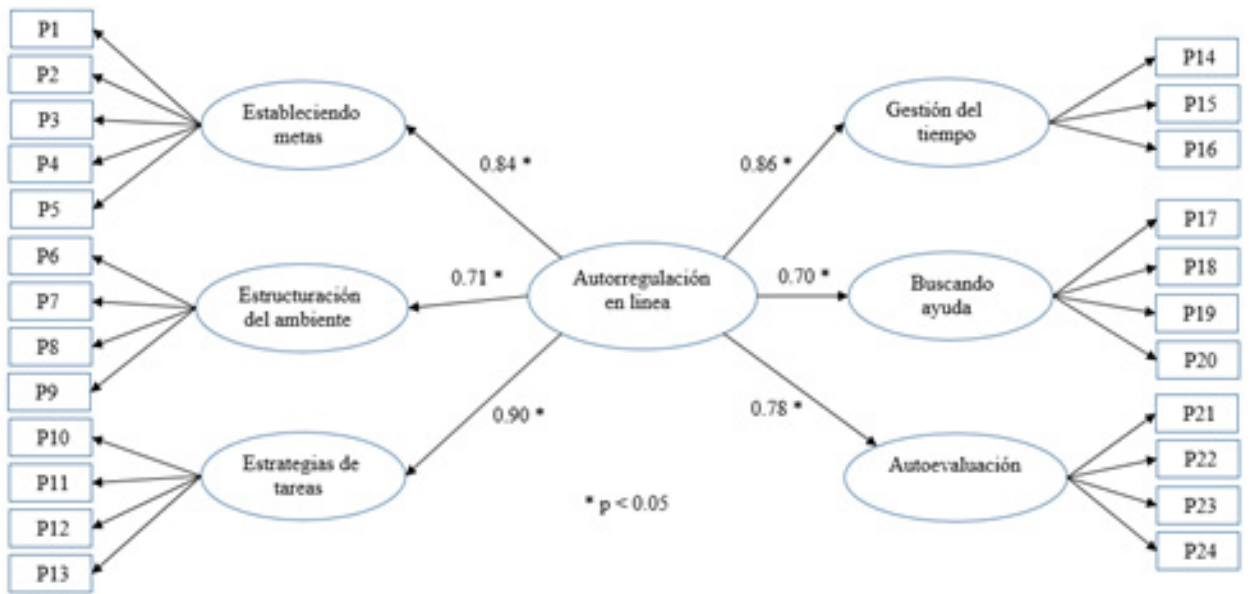

Fuente. Elaboración propia. 
Estudios Pedagógicos XLVI N²: 251-266, 2020

CUESTIONARIO DE AUTORREGULACIÓN DEL APRENDIZAJE EN LÍNEA (ONLINE SELF-REGULATED LEARNING QUESTIONNAIRE, OSLQ): ESTUDIO DE VALIDEZY FIABILIDAD DE LA VERSIÓN EN ESPAÑOL

Al realizar la prueba no-paramétrica de Kruskal-Wallis se obtiene como resultado:X2/ GL 3,5, RMSEA 0,07 y NNFI 0,98. Al comparar nuestro estudio con trabajos similares presentados en la tabla $\mathrm{N}^{\circ} 2$, concluimos que no existe diferencias estadísticamente significativas $(\mathrm{p}=0,763>0,05)$ en las medidas de bondad del ajuste para el modelo de análisis factorial confirmatorio considerado. Por lo tanto, los resultados logrados son equivalentes a los presentados en la literatura.

\section{DISCUSIÓN}

Nuestro estudio ha permitido obtener una versión en español del cuestionario OSLQ, validado a través de indicadores psicométricos. Para esto, a través del análisis factorial confirmatorio y consistencia interna se ratificaron los constructos propuestos por los autores Barnard et al., 2009.

El coeficiente de confiabilidad obtenido en la escala es 0,90 resultado consistente con lo informado por Barnard et al., que señala que las puntuaciones obtenidas demostraron una consistencia interna de 0,90 en estudiantes b-learning y 0,92 en estudiantes online (Barnard et al., 2009). Similares resultados se encontraron en otras investigaciones que utilizaron el OSLQ, los cuales se encuentran en un rango de 0,90 y 0,95 (Barnard et al., 2010b; MartínezLópez et al., 2017; Fung et al., 2018; Kilis y Yildirim, 2018).

$\mathrm{Al}$ examinar los resultados de la consistencia interna por subescala, los valores variaron entre 0,67 y 0,77 revelando suficiente fiabilidad de puntuación. Estos resultados se condicen con los obtenidos en la escala original, donde los valores de las subescalas variaron desde 0,67 hasta 0,90 , en la muestra de estudiantes matriculados en formato curso mixto y 0,87 a 0,96 en estudiantes en curso online. En las otras investigaciones, se encontró una mayor amplitud en los valores de consistencia interna oscilando entre 0,41 a 0,96. Lo anterior, puede ser consecuencia de los diferentes contextos socioculturales.

Si analizamos por subescala, la dimensión con menor consistencia interna corresponde a búsqueda de ayuda con un valor de 0,67, similar resultado fue obtenido por Barnard et al., con 0,69 en la muestra de estudiantes matriculados en formato curso mixto (Barnard et al., 2009). Dos investigaciones obtuvieron similares resultados en un rango de 0,60 a 0,63 (Kirmizi, 2014; Tabuenca et al., 2015; Kintu y Zhu, 2016). Lo anterior puede entenderse debido a que los estudiantes requieren búsqueda de apoyo en la educación a distancia (porque ellos están aislados de sus compañeros de clase y profesores (Holmberg, 1995; Hara y Kling, 2000, Wang y Newlin, 2002).

Sin embargo, estos resultados difieren de los encontrados por Barnard et al. con 0,96 en la muestra de estudiantes matriculados en formato curso online y en otros estudios con los siguientes resultados 0,92 y 0,96 (Barnard et al., 2009, 2010b; Onah y Sinclair, 2016).

La subescala Establecimiento de Metas obtuvo una consistencia de 0,69, resultados que no se condicen con los obtenidos por Barnard et al. con 0,86 en estudiantes matriculados en formato curso mixto (Barnard et al., 2009). Resultados levemente superiores con 0,76 y 0,79, se observan en las investigaciones de Kirmizi; Kilis y Yildirim (Kirmizi, 2014; Kilis y Yildirim, 2018).

En la subescala Estructura del Ambiente la consistencia alcanzada es 0,71 resultado inferior a los obtenidos por Barnard et al. en estudiantes online y mixto (Barnard et al., 2009). Similares resultados con 0,75 para Barnard et al. y 0,78 Tabuenca et al. (Barnard et al., 2010a; Tabuenca et al., 2015). 
Al analizar los resultados de la consistencia interna de la subescala Estrategias de tareas, se observa que el puntaje obtenido 0,71 es superior en el contexto de estudiantes b-learning (Barnard et al., 2009). En otras investigaciones se observa un amplio rango de resultados que varían entre 0,41 y 0,92 (Barnard et al., 2010a; Barnard et al., 2010b; Kirmizi 2014, Tabuenca et al., 2015; Onah y Sinclair, 2016; Lai y Hwang, 2016; Kintu y Zhu, 2016; Martínez-López et al., 2017; Fung et al., 2018; Kilis y Yildirim, 2018).

La subescala Gestión del tiempo obtuvo una consistencia interna de 0,76 , resultado similares a los obtenidos por Barnard et al. con 0,78 en estudiantes matriculados en formato curso mixto; 0,76 Barnard et al.; 0,71 Kirmizi; 0,76 Tabuenca et al.; 0,75 Kilis y Yildirim (Barnard et al., 2009; Barnard et al., 2010a; Kirmizi, 2014; Tabuenca et al., 2015; Kilis y Yildirim, 2018).

La subescala con mayor consistencia interna con un 0,77 corresponde a Autoevaluación, resultados que se condicen con los obtenidos por Barnard et al. con 0,78 en estudiantes matriculados en formato curso mixto, 0,72 y 0,73 Barnard et al.; Kintu y Zhu, respectivamente (Barnard et al., 2009, 2010a; Kintu y Zhu, 2016).

Los resultados no significativos encontrados en las subescalas pueden reflejar un desarrollo más lento de las habilidades autorreguladoras en los estudiantes en ambientes online o mixtos.

\section{LIMITACIONES Y FUTURAS INVESTIGACIONES}

Es importante señalar que la validación del OSLQ se realizó en una universidad pública y estatal chilena, que complementa la enseñanza con plataformas educativas, sin embargo, presenta un desarrollo inicial en la enseñanza exclusivamente online. Este contexto, acotó la muestra a estudiantes que cursaban asignaturas b-learning. Teniendo en consideración que este instrumento ha sido utilizado y validado en ambos ambientes de enseñanza, queda abierta la posibilidad de volver a validarlo en un contexto de modalidad online.

La relevancia de este estudio se sustenta en contar un instrumento validado que permita aplicarlo en otros contextos universitarios, inclusive otros países de habla hispana.

Adicionalmente, los estudiantes autorregulados se caracterizan por su participación activa en el aprendizaje desde el punto de vista metacognitivo, motivacional y comportamental, características que coinciden con las atribuidas a los alumnos de alto rendimiento. Sin embargo, para fomentar la autorregulación de estudiantes que utilizan plataformas educativas, es necesario documentar las conductas como el establecimiento de metas, estrategias usadas para afrontar las tareas, capacidad de autoevaluación del proceso, manejo del tiempo, regulación del ambiente de aprendizaje y la capacidad de pedir ayuda que intervienen en la capacidad de autorregulación del aprendizaje de forma fiable y válida, por lo que es importante desarrollar esta línea de investigación escasamente desarrollada a nivel latinoamericano. Complementariamente, una vez identificadas las conductas a desarrollar o fortalecer las que intervienen en el proceso de autorregulación, se pueden desarrollar estrategias de enseñanzas claras y concretas, sustentadas en la evidencia empírica.

Una forma de contribuir en esta línea de investigación en docencia es profundizando en los factores contextuales, motivacionales, estilos de aprendizajes, entre otros, que inciden en la capacidad de autorregulación de los estudiantes, a su vez poner a disposición 
Estudios Pedagógicos XLVI N² 2: 251-266, 2020

CUESTIONARIO DE AUTORREGULACIÓN DEL APRENDIZAJE EN LÍNEA (ONLINE SELF-REGULATED LEARNING QUESTIONNAIRE, OSLQ): ESTUDIO DE VALIDEZY FIABILIDAD DE LA VERSIÓN EN ESPAÑOL

un instrumento validado en español que permita medir la autorregulación en estudiantes universitarios.

Asimismo, se considera fundamental investigar la aplicación de este cuestionario a estudiantes en otro tipo de universidades, diferentes contextos socioeconómicos y culturales, con la finalidad de ver el comportamiento del instrumento y si se obtienen resultados similares a los presentados en este estudio. Esto generaría cuerpos de conocimientos que aportarían aún más en el desarrollo de este instrumento.

\section{CONCLUSIONES}

En este artículo se presentó los resultados de la traducción y validación al idioma español del Cuestionario Autorregulado del Aprendizaje en línea (OSLQ), en una muestra interdisciplinaria de 238 estudiantes universitarios. Los resultados obtenidos en las seis dimensiones del cuestionario presentaron índices de confiabilidad aceptable y consistente con los reportados por el autor original.

Asimismo, la versión original en idioma inglés fue cotejada con la versión en español, apreciándose su similitud y que la traducción del cuestionario mantenía el significado de los ítems originales.

Estos resultados permiten sustentar que este cuestionario puede comenzar a aplicarse con fines investigativos y de desarrollo académico en estudiantes universitarios.

\section{REFERENCIAS BIBLIOGRÁFICAS}

Allgood, W., Risko, V., Álvarez, M. y Fairbanks, M. (2000). Factors that influence study. En R. F. Flippo y D. C. Caverly (Coord.), Handbook of college reading and study strategy research, (pp. 201-219). NJ: LEA.

Ally, M. (2004). Foundations of educational theory for online learning. In The theory and practice of online learning, ed. T. Anderson, 15-44. Edmonton, CA: Athabasca University Press.

Azevedo, R. (2007). Understanding the complex nature of self-regulatory processes in learning with computer based learning environments: An introduction, Metacognition Learning, 2(2-3), 57-65.

Barnard, L., Lan, W., To, Y., Osland, V. \& Lai, S. (2009). Measuring self-regulation in online and blended learning environments. Internet and Higher Education, 12(1), 1-6.

Barnard, L., Osland, V. \& Lan, W. (2010a). Self-regulation across time of first generation online learners. ALT-J, Research in Learning Technology, 18(1), 61-70. DOI: 10.1080/09687761003657572

. (2010b). Profiles in Self-regulated Learning in the Online Learning Environment. The International Review of Research in Open and Distributed Learning, 11(1), 61-80. https://doi. org/10.19173/irrodl.v11i1.769

Bombardier, G. \& Beaton, C. (1993). Cross-cultural adaptation of health-re- lated quality of life measures. Literature review and proposed guidelines. Journal of Clinical Epidemiology, 46(12), 1417-1432.

Cunningham, C. A. \& Billingsley, M. (2003). Curriculum webs: A practical guide to weaving the Web into teaching and learning. Boston: Allyn and Bacon.

Fung, J., Yuen, M. \& Yuen, A. (2018). Validity Evidence for a Chinese Version of the Online Self-Regulated Learning Questionnaire with Average Students and Mathematically Talented Students, Measurement and Evaluation in Counseling and Development, 51(2), 111-124. DOI: 10.1080/07481756.2017.1358056 
Graham, C., Woodfield, W. \& Buckey H. (2013). A framework for institutional adaptation and implementation of blended learning in higher education. Internet and Higher Education, 18, 4-14.

Hara, N. \& Kling, R. (2000). Students Distress with a Web-based Distance Education Course: An Ethnographic Study of Participants' Experiences. Turkish Online Journal of Distance Education, 4(2). Recuperado de: http://www.slis.indiana.edu/CSI/wp00-01.html. Holmberg, B. (1995). Teoría y práctica de la educación a distancia. Nueva York, NY: Routhledge.

Holmberg, B. (1995). The sphere of distance-education theory revisited, (Servicio de reproducción Documentos ERIC N ${ }^{\circ}$ ED 386 578).

Hu, L. \& Bentler, P. (1999). Cutoff criteria for fit indexes in covariance structure analysis: conventional criteria versus new alternatives. Structural Equation Modeling, 6(1), 1-55.

Jaramillo, C. \& Chávez, J. (2015). Tic y Educación en Chile: Una revisión sistemática de la literatura. Nuevas Ideas en Informática Educativa TISE 2015.

Jöreskog, K. \& Sörbom, D. (2003). LISREL 8.54. Scientific Software International Inc., Chicago.

Kilis, S. \& Yildirim, Z. (2018). Online Self-regulation Questionnaire: Validity and Reliability Study of Turkish Translation. Çukurova Üniversitesi Eğitim Fakültesi Dergisi, 47(1), 233-245.

Kintu, M. \&. Zhu, Ch. (2016). Student Characteristics and Learning Outcomes in a Blended Learning Environment Intervention in a Ugandan University. The Electronic Journal of e-Learning, 14(3). Recuperado de: file:///C:/Users/Carmen\%20Mu\%C3\%B1oz/Downloads/ejel-volume14-issue3article505.pdf

Kirmizi, O. (2014). Self-regulated Learning Strategies Employed by Regular, Evening, and Distance Education English Language and Literature Students. The Anthropologist, 18(2), 447-460. Doi: 10.1080/09720073.2014.11891563

Lai, Ch. \& Hwang G. (2016). A self-regulated flipped classroom approach to improving students' learning performance in a mathematics course. Journal Computers \& Education, 100, 126-140. Doi: 10.1016/j.compedu.2016.05.006

Lan, W. Y., Bremer, R., Stevens, T. \& Mullen, G. (2004). Self-regulated learning in the online environment. Paper presented at the 2004 annual meeting American Educational Research Association, April 7-8, San Diego.

Lee, Y. \& Choi, J. (2011). A review of online course dropout research: Implications for practice and future research. Educational Technology Research and Development, 59(5), 593-618.

Leplège, A. \& Verdier, A. (1995). The adaptation of health status measures: methodological aspects of the translation procedure. En: Shumaker SA, Berzon R, eds. Quality of life: theory, translation, measurement \& analysis. Oxford: Rapid Communications of Oxford Ltd., 93-101.

Mac Callum, R., Browne, M. \& Sugawara, H. (1996). Power analysis and determination of simple size for covariance structure modeling. Psychological Methods, 18(2), 130-149.

Marsh, H. \& Hocevar, D. (1985). Application of confirmatory factor analysis to the study of selfconcept: First and higher-order factor models and their invariance across groups. Psychological Bulletin, 97(3), 562-582.

Martínez-López, R., Yot, C., Tuovila, I. \& Perera-Rodríguez, V. (2017). Online Self-Regulated Learning Questionnaire in a Russian MOOC. Computers in Human Behavior, 75, 966-974. Doi: https://doi.org/10.1016/j.chb.2017.06.015.

McManus, T. (2000). Individualizar la instrucción en un ambiente de aprendizaje hipermedia basado en la Web: linealidad, los organizadores previos, y los aprendices autorregulados. Journal of Interactive Learning Research, 11(2), 219-251.

Morán, L. (2012). Blended-learning. Desafío y oportunidad para la educación actual. Edutec, Revista Electrónica de Tecnología Educativa, (39). Recuperado de http://edutec.rediris.es/Revelec2/ Revelec39/blended_learning_desafio_oportunidad_educacion_actual.html

Norberg, A., Dziuban, C. \& Moskal, P. (2011). A time-based blended learning model. On the Horizon, 19(3), 207-216. dx.doi.org/10.1108/10748121111163913 
Estudios Pedagógicos XLVI Nº 2: 251-266, 2020

CUESTIONARIO DE AUTORREGULACIÓN DEL APRENDIZAJE EN LÍNEA (ONLINE SELF-REGULATED LEARNING QUESTIONNAIRE, OSLQ): ESTUDIO DE VALIDEZY FIABILIDAD DE LA VERSIÓN EN ESPAÑOL

Nunnally, J. (1978). Psychometric theory. 2nd ed. New York: McGraw-Hill.

Onah, D. \& Sinclair, J. (2016). Assessing Self-Regulation of Learning Dimensions in a Stand-alone MOOC Platform. International Journal of Engineering Pedagogy, 7(2). Recuperado de http:// online-journals.org/index.php/i-jep/article/view/6511/4451.

Pintrich, P. (2000). The role of goal orientation in self-regulated learning. En M. Boekaerts, P.R. Pintrich y M. Zeidner (Eds.), Handbook of self-regulation (pp. 451-502). San Diego, CA: Academic Press.

Ross, B. \& Gage, K. (2006). Global perspectives on blended learning, In C. J. Bonk \& C. R. Graham (Eds.), The handbook of blended learning - Global perspectives, local designs

(pp.155-167). CA: Pfeiffer - An Imprint of Wiley.

Schunk, D. H. \& Zimmerman, B. J. (Eds.). (1994). Self-regulation of learning and performance: Issues and educational applications. Hillsdale, NJ, US: Lawrence Erlbaum Associates, Inc.

Schunk, D. H. \& Zimmerman, B. J. (1998). Selfregulated learning: From teaching to self-reflective practice. New York: Guilford Press.

Schunk, D. H. (2001). Social cognitive theory and self-regulated learning. In B. J.

Tabuenca, B., Kalz, M., Drachsler, H. \& Specht, M. (2015). Time will tell: the role of mobile learning analytics in self-regulated learning. Computers \& Education, 89, 53-74.

Vaughan, N., Cleveland-Innes. M. \& Garrison, D. (2013). Teaching in Blended Learning Environments: Creating and Sustaining Communities of Inquiry. Edmonton: AU Press.

Wang, A. \& Newlin, M. (2002). Predictors of Web-Student Performance: the role of self- efficacy and reasons for taking an on-line class. Computers in Human Behavior, 18(2), 151-163.

Zimmerman, B. J. (1990). Self-regulated Learning and academic achievement: An overview. Educational Psychologist, 25(1), 3-17.

Zimmerman, B. J. (1998). Academic studying and the development of personal skill: A self-regulatory perspective. Educational Psychologist, 33(2-3), 73-86.

. (2001). Theories of self-regulated learning and academic achievement: An overview and analysis, en Self-regulated learning and academic achievement: Theoretical perspectives, $2^{\mathrm{a}} \mathrm{ed}$, Lawrence Erlbaum Associates, pp. 1-37, Mahwah NJ: USA.

Zolotykhin, S. A. (2015). The use of learner-focused approach when designing of massive open online courses. Sovremennye Issledovaniya Sotsialnykh Problem, (8). http://dx.doi. org/10.12731/2218-7405-2015-8-19. 